\title{
EL SINDROME DEL QUEMADO EN EL PERSONAL SANITARIO Y LA SATISFACCIÓN DEL PACIENTE
}

\section{BURNOUT SYNDROME IN HEALTH WORKERS AND PATIENT SATISFACTION}

Rocío Cano Sánchez, Zoraida Ciruela Palomino, Irene Gómez Gómez, Ángel Mata Fernández, Carolina Montalvo Vico y Héctor Olmedo Sánchez. Universidad de Granada.

\section{Resumen}

El presente estudio tiene dos objetivos, por un lado conocer e identificar el síndrome del quemado en el personal médico de tres hospitales de Granada y por otro lado conocer la satisfacción de los pacientes a la hora de ser atendidos. Para tales objetivos se ha seleccionado una muestra de un total de 57 trabajadores sanitarios a los cuales se les administró el Maslach Burnout Inventory (MBI) y 50 pacientes a los que se les administró la Encuesta de Opinión sobre la Atención Hospitalaria (SERVQHOS). Los principales resultados muestran diferencias significativas en la escala de cansancio emocional pero sólo entre enfermeros y médicos, además se han encontrado diferencias significativas en las escalas de cansancio emocional y despersonalización entre los tres hospitales, y por último en cuanto a las pacientes no se han encontrado diferencias significativas ni en sexo ni edad.

Palabras Clave: satisfacción, personal médico, paciente, síndrome de estar quemado.

\section{Abstract}

The present study has two aims, in one hand try to know and identify the burnout syndrome in medical personnel in three Granada's hospitals, on the other hand the study try to know the patients' satisfaction when they are being looked after. For this two aims we have selected a sample of 57 medical personnel who have taken Maslach Burnout Inventory (MBI) and 50 patients who have taken the Encuesta de Opinión sobre la Atención Hospitalaria (SERVQHOS). The main results showed significant differences in emotional exhaustion scale but only between nurses and doctors, in addition we have found significant differences in emotional exhaustion scale and depersonalization scale between the three hospitals, and finally related with patients we haven't found significant differences in sex and age.

Keywords: satisfaction, medical personnel, burnout syndrome, patients. 


\section{INTRODUCCIÓN}

La Ley General de Sanidad establece como principios generales la eficacia, la economía, la racionalización, la organización, la coordinación y la integración de los recursos sanitarios públicos, para hacer efectivas las prestaciones sanitarias y mantener altos niveles de calidad debidamente evaluados y controlados (Ley 14/1986, de 25 de Abril, General de Sanidad; Real Decreto $63 / 1995$, de 20 de enero, sobre ordenación de prestaciones sanitarias del Sistema Nacional de Salud).

Por ello la evaluación del sistema de organización de los servicios sanitarios se ha convertido en una tarea esencial de los sistemas de salud y se ha incorporado a los organismos encargados de evaluar tecnologías sanitarias. La satisfacción de los pacientes es clave en la mejora de la calidad asistencial y también se utiliza como medida de los resultados de los cuidados y se ha asociado con aspectos relacionados con la salud tan importantes como la adhesión al tratamiento y las recomendaciones de los profesionales, el cambio de proveedor, e incluso la mejora de la salud (Delgado, 1995; Prieto, 1999). A pesar de su reconocida importancia, la satisfacción del paciente con los servicios de salud, no es suficientemente investigada en España y, cuando se hace, son empleados métodos, enfoques e instrumentos muy heterogéneos entre sí (Clíments, 2003).

La satisfacción puede definirse como "la medida en que la atención sanitaria y el estado de salud resultante cumplen con las expectativas del usuario"(Corbella 1990). El grado de calidad de los servicios ofertados en un sistema sanitario está directamente relacionado con el nivel de satisfacción de los profesionales que trabajan en él y en su vinculación con la motivación (García, 1995; Olivar, 1999).

En un estudio sobre la satisfacción de 328 pacientes sobre la atención del personal médico del Subsistema de Urgencias en Atención Primaria de Salud (SUAPS), los elementos que más se relacionaron con la satisfacción fueron el tiempo de espera, el ambiente adecuado y el trato recibido (Chang, 1999). En el mismo se encontraron diferencias entre sexos -las mujeres manifiestan una mayor insatisfacción- y entre edades -los de mayor edad son más críticos con los servicios sanitarios-.

En las últimas dos décadas ha habido un creciente reconocimiento público de las limitaciones de la medicina (Feletti, 1986). Donabedian (1982) afirma que la satisfacción es un componente integral y esencial para la estimación de la calidad de la atención médica.

El síndrome del quemado, que se conceptualiza como una respuesta al estrés laboral crónico, parece afectar en mayor o menor medida a un colectivo profesional importante. No hay un consenso global para la explicación de este síndrome de desgaste profesional. Una de las teorías con más aceptación es la proporcionada por Maslach, en la que el síndrome estaría caracterizado por tres dimensiones interrelacionadas: el cansancio emocional, la despersonalización y la realización personal (Férnandez, 2000; Maslach, 1986). 
La relación entre satisfacción laboral y desgaste profesional es estrecha. Bajos niveles de satisfacción se asociaron con altas puntuaciones en las escalas de cansancio emocional y despersonalización, y bajas puntuaciones en realización personal (Sobrequés, 2003).

Teniendo en cuenta las argumentaciones anteriores y estudios realizados, nuestros objetivos principales han sido dos, por un lado identificar y analizar el Síndrome de quemado (Burnout) en el personal sanitario, y por otro lado, comprobar la satisfacción de los pacientes a la hora de ser atendidos, en sus distintas dimensiones, centrándonos en hospitales de Granada capital (Hospital Universitario San Cecilio, Hospital Materno Infantil Virgen de las Nieves, Hospital de Rehabilitación y Traumatología Virgen de las Nieves).

H1: Los médicos muestran menor cansancio emocional y despersonalización que los enfermeros y auxiliares.

$\mathrm{H} 2$ : Los resultados del cuestionario $\mathrm{MBI}$ estarán igualados en los tres centros hospitalarios.

H3: La satisfacción (global, objetiva y subjetiva) será menor en pacientes mujeres.

H4: La satisfacción global se relacionará inversamente con la edad.

\section{MÉTODO}

\section{Participantes}

La población que ha participado en este estudio se puede dividir en dos grupos, por un lado, personal sanitario, formado por 11 médicos, 26 enfermeros, 17 auxiliares de enfermería y 3 celadores, haciendo un total de 57 personas, de los cuales 6 son hombres y 51 mujeres. Todos ellos trabajadores de tres centros médicos de Granada (Hospital Universitario San Cecilio, Hospital Materno Infantil Virgen de las Nieves, Hospital de Rehabilitación y Traumatología Virgen de las Nieves). Por otro lado, el siguiente grupo consta de 50 pacientes ( 25 hombres y 25 mujeres) que debían cumplir el requisito de acudir o haber acudido en el último año a los centros antes mencionados. La edad de los sujetos en ambos grupos se encuentra entre 18-65 años.

\section{Materiales}

Para el primer grupo de participantes (personal sanitario) se utilizó el Maslach Burnout Inventory (MBI), que se trata de un cuestionario de 22 ítems con 7 opciones de respuesta (escala Likert de 0 a 6) y posee tres subescalas que miden el cansancio emocional (sentimiento del sujeto respecto a encontrarse saturado emocionalmente por el trabajo), despersonalización (respuesta fría e impersonal hacia los pacientes) y realización personal (sentimientos de competencia y eficacia en la realización del trabajo). Las subescalas de cansancio emocional y despersonalización indican mayor desgaste a mayor puntuación, mientras que la subescala de realización personal funciona en sentido inverso, indicando mayor desgaste las puntuaciones bajas. En el 
presente estudio se obtuvo un coeficiente de fiabilidad alfa de Cronbach de .81 en cansancio emocional, .31 en despersonalización y .41 en realización personal.

Para el segundo grupo (pacientes) se empleó la Encuesta de Opinión sobre la Calidad de la Atención Hospitalaria (SERVQHOS) que es uno de los instrumentos más utilizados en el sector servicios para evaluar el nivel de satisfacción y la calidad percibida por los clientes. Consta de 19 ítems, divididos en dos subescalas que miden la satisfacción objetiva y subjetiva. Además se evalúa el grado de satisfacción global de los pacientes. En relación a la fiabilidad, en este estudio se obtuvo un coeficiente de Cronbach de .96 en satisfacción global, .86 en satisfacción objetiva y .84 en subjetiva.

\section{Procedimiento}

Acudimos a los 3 centros hospitalarios mencionados con anterioridad para pasar el cuestionario $\mathrm{MBI}$ a la mayor parte de personal sanitario posible y dispuesto a participar, y a su vez, repartimos el cuestionario SERVQHOS a una muestra aleatoria de personas que acuden en el presente o han acudido en el último año a uno de estos centros. Una vez pasados los cuestionarios recurrimos al análisis estadístico de los datos mediante el programa SPSS.

\section{RESULTADOS}

La presentación de los resultados se dividirá en dos estudios, por un lado el referente al personal sanitario y por otro lado, el referente a los pacientes.

\section{Estudio 1}

Nuestra intención en este primer estudio es comparar las profesiones (médico, enfermero y auxiliar de enfermería), con las diferentes escalas medidas por el cuestionario $\mathrm{MBI}$ (cansancio emocional, despersonalización, y realización personal) por un lado, y por otro, comparar además estas mismas escalas, en función de la pertenencia a un centro hospitalario u otro (Materno Infantil, Clínico y Traumatología). Con respecto a la comparación entre profesiones, no se tuvo en cuenta a los celadores, ya que la muestra era escasa.

Con el objetivo de analizar si existían diferencias entre los participantes en función de su profesión en las puntuaciones obtenidas en las distintas escalas, se realizó una $t$ de student para muestras independientes.

Los resultados mostraron diferencias significativas, $t(35)=3.76 ; p<.001$ entre médicos y enfermeros, exclusivamente en la escala de cansancio emocional, siendo mayor en los últimos. No se obtuvieron diferencias significativas comparando médicos con auxiliares y enfermeros con auxiliares, en ninguna escala. 
Gráfica 1. Comparación entre las puntuaciones del MBI de los diferentes grupos del personal sanitario.

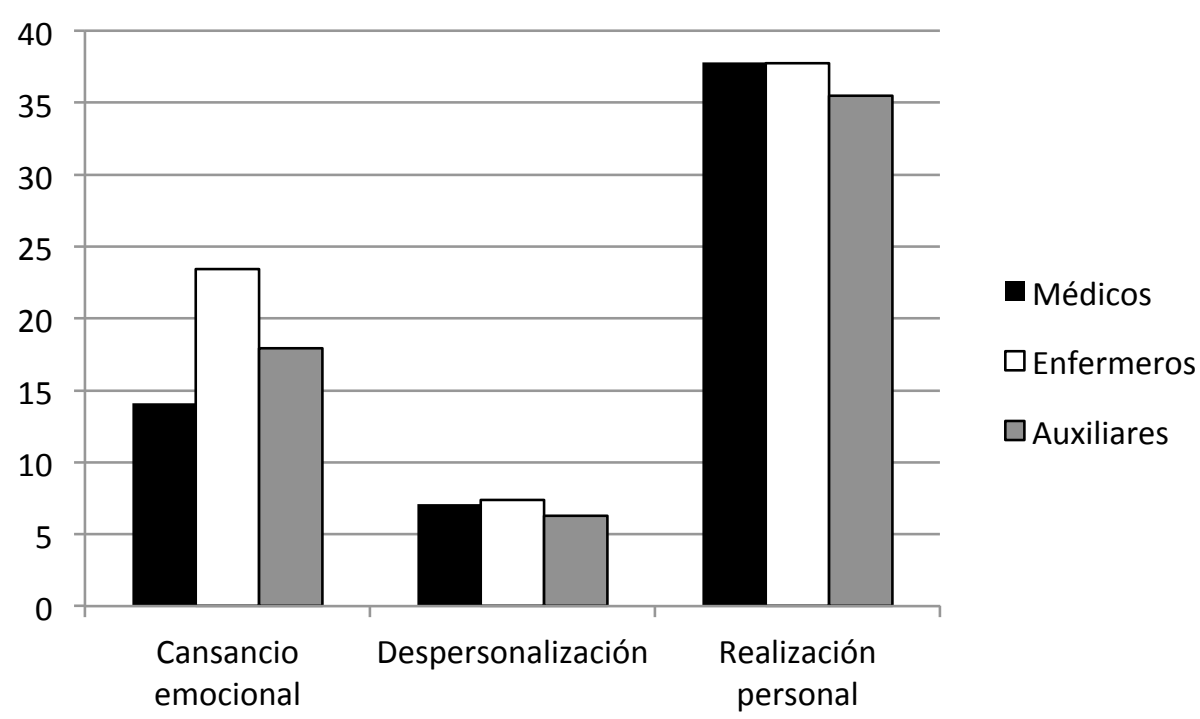

Con la finalidad de analizar si existían diferencias entre el centro hospitalario en el que trabajan y las puntuaciones obtenidas en las distintas escalas, se realizó una $t$ de student para muestras independientes.

Los resultados mostraron diferencias significativas en las escalas de cansancio emocional y despersonalización $t(42)=3,66 ; p<.001$ y $t(42)=2,87 ; p=.006$ respectivamente entre los hospitales Materno Infantil y Clínico, siendo mayores en el primero. También hubo diferencias significativas en la escala de cansancio emocional $t(36)=4.60 ; p<.001$ en los hospitales Materno Infantil y Traumatología, siendo mayor en el primero en este caso también. Con respecto a los hospitales Clínico y Traumatología se encontraron diferencias significativas en la escala de despersonalización $t(28)=2.2 ; p=.040$, siendo mayor en éste último.

Gráfica 2. Comparación entre las puntuaciones del MBI de los diferentes centros hospitalarios.

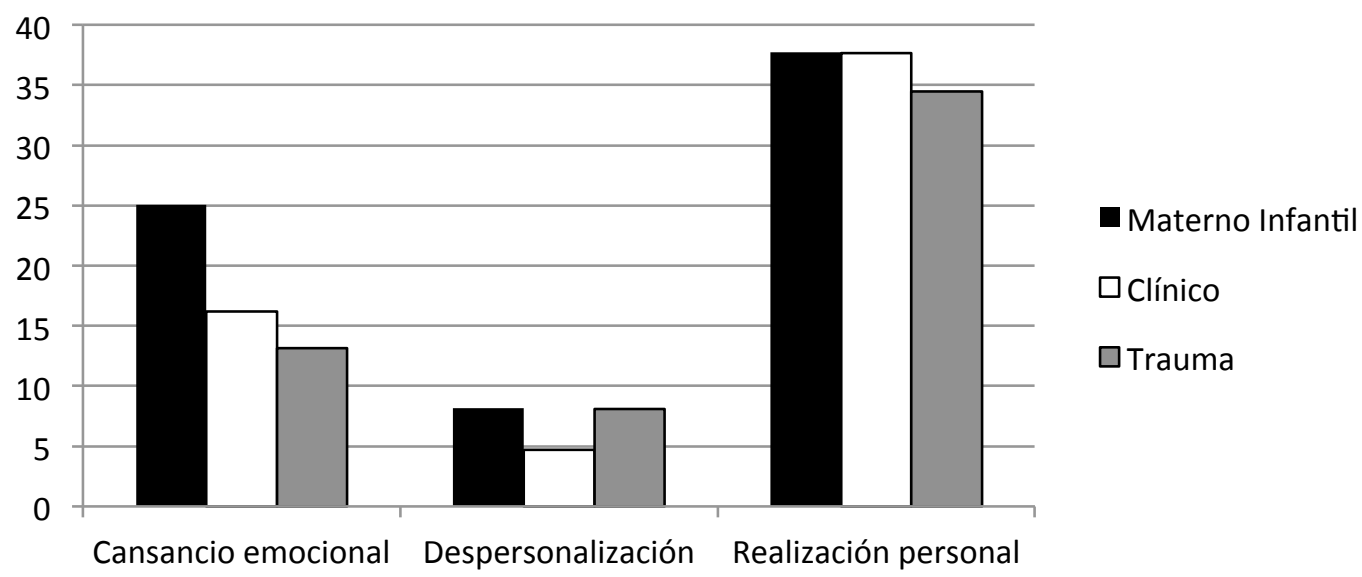




\section{Estudio 2}

En este estudio pretendemos comparar las distintas dimensiones de satisfacción (objetiva, subjetiva y global) entre el sexo de los pacientes por un lado, y su edad por otro.

Con el objetivo de analizar si existían diferencias en las puntuaciones obtenidas en las distintas dimensiones en función del sexo de los participantes, se realizó una $t$ de student para muestras independientes.

Tabla 1. Resultados t de student, media y desviación típica de cada escala en función del sexo.

\begin{tabular}{lllcl}
\hline & sexo & N & Media & DT \\
\hline \multirow{2}{*}{ satifacciónGLOBAL } & hombre & 25 & 2.84 & 0.68 \\
& mujer & 25 & 2.84 & 0.68 \\
\hline \multirow{2}{*}{ SatisfaccionSubjetiva } & hombre & 25 & 3.20 & 0.57 \\
& mujer & 25 & 3.09 & 0.67 \\
\hline \multirow{2}{*}{ SatisfaccionObjetiva } & hombre & 25 & 2.95 & 0.54 \\
& mujer & 25 & 2.95 & 0.64 \\
\hline
\end{tabular}

Los resultados han mostrado que no existen diferencias significativas en ninguna dimensión. Incluso se han encontrado puntuaciones exactamente iguales en todas las dimensiones de la encuesta, tanto en hombres como en mujeres.

Con el fin de comprobar la posible relación entre la satisfacción y la edad, se llevó a cabo un análisis de correlación de Pearson bivariado, teniendo en cuenta los distintos tipos de satisfacción.

Los resultados obtenidos muestran que la edad no está relacionada con la satisfacción. Sin embargo, correlacionan significativa y positivamente la escala de satisfacción subjetiva con la global $(r=.42 ; p<.01)$ e igualmente la escala objetiva con la global $(r=.46 ; p<.01)$.

\section{DISCUSIÓN}

El objetivo del trabajo ha sido, por un lado describir el estado de distintos trabajadores sanitarios en tres centros de la provincia de Granada, en relación al síndrome de burnout, y por otro lado describir la satisfacción de pacientes que acuden normalmente a dichos centros.

En cuanto a la primera hipótesis propuesta sólo se obtuvieron diferencias significativas en relación a médicos y enfermeros, en la escala de cansancio emocional, siendo estos últimos los que se encuentran más afectados. 
Posiblemente debido al tamaño de la muestra no se obtuvieron resultados significativos en cuanto a auxiliares.

En relación a la segunda hipótesis acerca del grado de burnout en los distintos centros, se ha encontrado que el hospital Materno Infantil presenta mayor cansancio emocional que el Clínico y Traumatología, y mayor grado de despersonalización con respecto al Clínico. Por otro lado, Traumatología presenta mayor despersonalización que el Clínico. Estas diferencias pueden deberse a la afluencia de pacientes que tiene cada hospital para determinadas consultas, o a la especialidad de los trabajadores, como por ejemplo en Oncología Infantil, presente en el hospital Materno Infantil.

La importancia de estas dos hipótesis reside en que, se puede estimar los profesionales y los hospitales en los que se presenta más desgaste profesional. Con ello, podría mejorarse la satisfacción y a su vez, el trato con el paciente. Diversos estudios, como el de Cebrià et al (2000) o el de González (1998), han analizado los factores asociados a dicho desgaste profesional pero, su relación con la satisfacción ha sido poco explorada en el ámbito nacional.

Según nuestra tercera hipótesis las mujeres pacientes deberían tener una puntuación menor en satisfacción tanto global como objetiva y subjetiva. Así lo indican otros estudios argumentando además que ellas son las que solicitan asistencia sanitaria con más frecuencia (Chang, 1999). Sin embargo según los resultados esta hipótesis no se cumple debido a que no hay diferencias significativas según la prueba $\mathrm{T}$ de student en ninguna dimensión entre sexos. Esto puede deberse a sesgos en el estudio como el tamaño de la muestra que no es demasiado extensa ( 25 hombres y 25 mujeres) por lo que en futuras investigaciones se habrá de seleccionar una muestra mayor.

En cuanto a la relación entre edad y satisfacción no se ha encontrado que exista correlación. Esto contrasta con que los grupos de 40 a 49 años son los más críticos peyorativamente hacia la sanidad (Ruiz, 1990). Si bien, si existe entre los distintos tipos de satisfacción una correlación, encontrando que a mayor satisfacción global, mayor satisfacción objetiva y subjetiva.

En futuros estudios podría resultar interesante comparar el síndrome de burnout entre hombres y mujeres sanitarios, ya que en este caso no se ha tenido en cuenta por el evidente mayor número de mujeres de nuestra muestra. Además, del mismo modo, también podrían existir diferencias entre las distintas especialidades médicas entre dicho personal sanitario. Esto es importante para el conocimiento sobre la especialidad en la que se podría comenzar a prevenir o tratar el síndrome en aras de una mejora de la satisfacción del trabajador que repercute en la calidad de la atención a los pacientes.

\section{REFERENCIAS}

Cebrià J, Segura J, Corbella S, Sos P, Comas O, García M, et al. (2000) Rasgos de personalidad y burnout en médicos de familia. Atención Primaria, 27, 459-68. 
Chang, M, Alemán, M.C., Cañizares, M. y Ibarra, A.M. (1999) Satisfacción de los pacientes con la atención médica. Revista Cubana de Medicina General Integral, 15(5), 541-547.

Clíments, G. D. y Aguirre-Jaime, A. (2003).Enfermera amable, paciente satisfecho. Validación de una nueva escala de la satisfacción de los usuarios. Enfermería clínica, 13(1), 7-15.

Corbella, A. y Saturno, P. (1990) La garantía de la calidad en atención primaria de salud. Madrid: Instituto Nacional de Salud, Secretaría General (pp 397-399).

Delgado A. (1997) Factores asociados a la satisfacción de los usuarios. Cuadernos de Gestión, 3(2):90-101.

Donabedian, A. (1997). The quality of care.Archives of pathology \& laboratory medicine 121, 11.

Feletti, G., Firman, D., ySanson-Fisher, R. (1986).Patient satisfaction with primary-care consultations. Journal of behavioral medicine, 9(4), 389-399.

Fernández M.I., Moinelo, A., Villanueva, A., Andrade, C., Rivera, M., Gómez, J.M., et al (2000). Satisfacción laboral de los profesionales de atención primaria del área 10 del Insalud de Madrid. Revista Española de Salud Pública, 74(1),139-47.

García, S. y González, J. A. (1995). Factores de motivación de los profesionales de la salud en Atención Primaria. FMC, 2, 1

González, P., Suberviola, R. y González, J.F. (1998). Prevalencia del síndrome de burnoutr o desgastge profesional en los médicos de atención primaria. Atención Primaria, 22, 580-584.

Maslach, C., y Goldberg, J. (1999). Prevention of burnout: New perspectives. applied and Preventive Psychology, 7(1), 63-74.

Mira, J.J., Aranaz, J., Rodríguez-Marín, J., Buil, J.A., Castell, M., Vitaller, J. (1998). SERVQHOS: un cuestionario para evaluar la calidad percibida de la asistencia hospitalaria. Medicina Preventiva, 1(4), 12-18.

Olivar, C., González, S. y Martínez, M.M. (1999). Factores relacionados con la satisfacción laboral y el desgaste profesional en los médicos de atención primaria de Asturias. Atención Primaria, 24, 352-359.

Prieto, M.A., March, J.C. y López F. (1999). Calidad percibida por usuarios de centros de salud y de aseguradoras privadas. Atención Primaria, 24, 259-266.

Ruiz Chávez, M. (1990) Bases para la evaluación de la calidad de la atención médica en las unidades médicas del sector salud. Salud Pública Mexicana, 32(2), 156-69.

Sobrequés, J., Cebriá, J., Segura, J., Rodríguez, C., García, M., y Juncosa, S. (2003). La satisfacción laboral y el desgaste profesional de los médicos de atención primaria. Atención Primaria, 31(4), 227-233. 